$$
\text { Conf-9105136 }
$$

\title{
EVOLUTION OF DEFECT CLUSTER DISTRIBUTIONS DURING IRRADIATION*
}

\author{
H. WIEDERSICH \\ Materials Science Division \\ Argonne National Laboratory \\ Argonne, Mlinois, USA.
}

ANL/CP- 72655

DE91 013405

May 1991

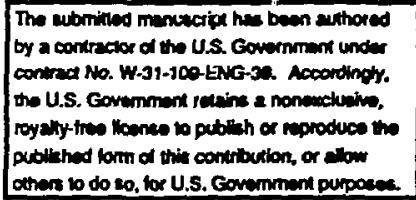

\section{DISCLAIMER}

\begin{abstract}
This report was preparod as an account of wrik sponsored by an agency of the United States Government. Neither the United States Government nor any agency thereof, sor any of their employees, makes any warranty, express or implied, or assumes any legal liability or responsibility for the accuracy, completeness, or usefulness of any information, apparatus, product, or process disclosed, or represents that its use would not infringe privately owned rights. Reference herein to any specific commercial product, process, or service by trade name, trademark, manufacturer, or otherwise does not necessarily constitute or imply its endorsement, recommendation, or favoring by the United States Governinent or any agency thereof. The views and opinions of authors expressed herein do not necessarily state or reflect those of the United States Government or any agency thereof.
\end{abstract}

Invited paper submitted to the "International Conference on Physics of Irradiation Effects in Metals", to be held in Siofok, Hungary, May 20-24, 1991.

*Work supported by the U. S. Department of Energy, BES-Materials Sciences, under Contract W-31-109-Eng-38. 


\title{
EVOLUTION OF DEFECT CLUSTER DISTRIBUTIONS DURING IRRADIATION*
}

\author{
H. WIEDERSICH \\ Materials Science Division, Argonne National Laboratory, Argonne, Mlinois, \\ USA.
}

\begin{abstract}
Defects produced by irradiation are often strongly spatially correlated. Energetic primary recoil atoms produce cascades with vacant lattice sites predominantly in the central regions, and interstitial atoms at the periphery. $A$ fraction of the defects produced form clusters during cascade events. Because of their spatial distribution more mobile interstitial atoms than vacancies are released into the matrix following the cascade events. Populations of vacancy and interstitial clusters evolve towards quasi-steady-state distributions. As long as vacancy clusters are thermally moderately stable, i. e., at temperatures below rapid self diffusion, an excess interstitial flux persists in the matrix; as a consequence, vacancy clusters formed in cascades shrink, and interstitial clusters grow. We examine the evolution of these cluster distributions, and their effects on sink strength and radiation-enhanced diffusion, as function of dose for irradiations at moderate temperatures. At temperatures at which thermal evaporation from vacancy clusters can be neglected, the evolution of the cluster densities is characteristic of the primary recoil spectrum, but is independent of temperature or dose rate. The sink strength, radiation-enhanced diffusion, as well as other consequences of the evolving distributions such as the imbalance of the interstitial and vacancy fluxes in the matrix, approach slowly-changing quasisteady-state values in a fraction of one displacement per atom, although certain details of the cluster distributions, e. g., the development of large interstitial loops, require several dpa's to approach steady state values.

*Work supported by the U. S. Department of Energy, Basi: Energy SciencesMaterials Sciences under Contract \#W-31-109-ENG-8
\end{abstract}




\section{Introduction}

It has long been recognized that the defect production calculated from codes based on straightforward binary collision approximations and the Kinchin and Pease formula overestimates the number of individual point defects produced by energetic particle bombardment see, e. g., references [1-4]. Even if one takes spontaneous recombination of defects produced within cascades in close proximity to each other into account, the calculated number of surviving defects exceeds significantly the experimentally observable numbers at elevated temperatures especially for irradiations with heavy ions and with high energy neutrons. $[5,6]$ The knowledge of the fraction of defects that survive and undergo long range migration is of particular importance to the interpretation and prediction of microstructural changes occurring in materials during irradiations with neutron and energetic charged particles, since a number of these changes, such as radiation-induced precipitation and void swelling, require the migration of alloying elements and/or defects over considerable distances.

Transmission electron microscopy observations have shown that a large fraction of the deferts produced in energetic cascade events are observed as clusters in the form of vacancy dislocation loops, stacking fault tetrahedra and, to a lesser extent, as interstitial dislocation loops (see, e. g., Black et al. [7] Kiritani et al. [8]). A number of treatments concerned with the survival of individual or "free" defects, potentially undergoing long range migration, have dealt with these clusters in a more or less heuristic manner (e. g., Heinisch [9], Wiedersich [10])

In this paper we present a simple treatment, in the framework of rate theory [11], of the evolution of defect cluster distributions during irradiations in which the defects are produced in cascades. Bullough, Eyre and Krishan have proposed a way of including the effects of small vacancy loops on void swelling. [12] Bullough and Quigley have shown that void swelling of M316 steel can be predicted rather precisely for widely varying irradiation conditions when one applies the model properly. [13] However, their approach to the small defect cluster problem has been largely ignored in the general treatments of microstructural changes probably, because of the complexity of the model which includes the varying bias effects of several sink types.

Recently, Woo and Singh considered the effects of spontaneous clustering of interstitials and of vacancies in cascades. [14] They pointed out that clustering of interstitials can lead to a "production-bias" term and to an anomalously large woid swelling rate if the vacancy clusters formed in 
cascades are highly unstable with regard to thermal decomposition. Dut the interstitial clusters are thermally stable.

The present treatment is an extension of previous work on steady state cluster distributions which ultimately develop during prolonged irradiations [15]. Here, we explore the evolution towards steady state. As in the previous work, we focus on situations where vacancy clusters are thermally stable or moderately stable. The approximate temperature range for which the treatment should be applicable is from stage III, above which vacancies become thermally mobile up to near stage $V$, when vacancy clusters of significant size anneal out thermally; see,e.g., [16] . This temperature range corresponds in fcc metals from $0.2-0.25 \mathrm{~T}_{\mathrm{m}}$ to $0.4-0.5 \mathrm{~T}_{\mathrm{m}}$ depending on the metal ( $\mathrm{T}_{\mathrm{m}}$ is the absolute melting temperature). For simplicity, we ignore bias effects since the treatment is meant to be applied to phenomena such as radiation-induced segregation and radiationenhanced diffusion, where bias effects are not central in contrast to void swelling and radiation creep.

\section{Model description.}

Irradiation with energetic particles almost invariably produces defects not only in form of isolated Frenkel pairs but also in the form of clusters of closely spaced defects in cascades. In fact, the majority of defects is created in cascades in the case of irradiations with heavy ions and, even more so, with high energy neutrons. As a consequence, a significant fraction of the point defects created in cascades are lost by mutual recombination or form clusters of like defects during the cascade life-time $\left(-10^{-12} \mathrm{~s}\right)$. It has been established experimentally (see, e. g., Black et al. [7], Kiritani et al. [8]) that vacancies form dislocation loops and stacking fault tetrahedra. Evidence for the formation of interstitial dislocation loops, often in clusters of several loops, during $14 \mathrm{MeV}$ neutron irradiations of copper, e.g., has been presented by Yoshida et al. [17]. Molecular dynamics simulations (see, e. g., Guinan and Kinney [18], Diaz de la Rubia et al. [19]) indicate that vacancies cluster predominantly in the central regions of cascades, whereas interstitials form clusters at the periphery.

For the present purpose we will assume that the primary knock-on spectrum produces clusters at a rate $K_{i c l, v c l}$ containing $n_{i o, v o}$ interstitials (subscript $i$ ) or vacancies (subscript $v$ ). In reality there will be a distribution of cluster sizes, but for simpiicity we will assume here that they are all of the same, average size. Both $\mathbf{K}$ and $\mathbf{n}$ will be different for interstitials and for vacancies. The clusters will shrink or grow depending on the net condensation rate of defects on clusters which, in turn, will be determined by the effective medium concentration [20] of mobile interstitials, $c_{i}$, and vacancies, $c_{v}$, and by the cluster size dependent 
evaporation rate of defects. The cluster type that experiences shrinkage will tend towards a steady state cluster concentration; the type that grows will become ultimately a part of the "network" dislocation density. We note here that it is not inconceivable that both types of clusters shrink, e. g., at sufficiently high temperatures, when evaporation of defects from small clusters may be dominant even for interstitial clusters.

At temperatures of interest here, i. e., at which both types of point defects are mobile one can expect that the material under irradiation approaches a quasisteady-state. The relaration times for approaching such a quasi-steady-state for a species depend on the species of interest, e.g., point defects, defect clusters, dislocation loops. Although the microstructure as a whole continues to evolve, and, hence, the quasi-steady-state for every species changes with the microstructure, the concept of a quasi-steady-state is quite useful for species with relatively short relaxation times. Generally, we can expect that quasi-steadystates are reached in sequence for interstitials, for vacancies, for cascade produced defect clusters that shrink, followed by those clusters which grow and get incorporated into the dislocation network and, finally, the network itself. The void number density seems to have a similar relaxation time as that for the network, whereas processes that require long range transport of alloying elements, such as radiation induced precipitation, have relaxation times which may too long to make a quasi-steady-state treatment useful.

Our approach will be to determine the quasi-steady state concentrations of mobile vacancies and interstitials in the effective medium by a set of rate equations which takes into account that the rate of freely migrating defects is reduced from the number of initially produced defects (displacements per atom) not only by mutual annihilation, but also by clustering of like defects during the cascade event. Vacancy clusters are assumed to be formed in the central regions of cascades, whereas interstitial clusters nucleate during the cascade event in peripheral regions. Furthermore, preexisting defect clusters will be redissolved if located within the central region of a subsequent energetic cascade. This region can be taken to be the equivalent to the "molten zone" [19] or region in which the deposited energy density during the collisional phase exceeds, say, the latent heat of melting. The crystallinity of the material surrounding the molten zone is preserved during the cascade event; therefore, we assume that the net number of defects redissolved from clusters in this region is preserved and is incorporated into the newly formed vacancy cluster(s) of the nascent cascade.

Of the defects formed during a cascade event, $N_{0}$, fractions $f_{v}$ and $f_{i}$ will escape from the immediate vicinity of the cascades and will enter into the general background population of mobile defects in the effective medium. The remainder will either recombine, fraction $f_{r}$, which is equal for both type of defects, or form 
clusters, fractions $f_{\mathrm{vcl}}$ and $\mathrm{f}_{\mathrm{icl}}$ for vacancies and interstitials, respectively. We note here that only three of the five fractions are independent since the relations 1 $=f_{v}+f_{v c l}+f_{r}$ and $1=f_{i}+f_{i c l}+f_{r}$ must hold. The fractions will depend on the cascade size, $N_{0}$, with $f_{V}$ and $f_{i}$ decreasing, and $f_{r}, f_{v c l}$ and $f_{i c l}$ increasing with increasing cascade size. The clusters themselves are corsidered as immobile and their sink strengths are included into the total sink strength, $\mathrm{p}_{v}$ and $\mathrm{p}_{\mathrm{j}}$.

With these definitions we can write down the stanciard rate equations $[11,21]$ for the steady state concentrations of vacancies, $c_{v}$, and interstitials, $c_{j}$, as:

$$
\frac{\partial c_{V}}{\partial t}=0=K f_{V}+K{ }_{V}^{t h}-A c_{i} c_{v}-p_{v} v_{v} c_{v}
$$

and

$$
\frac{\partial c_{i}}{\partial t}=0=K f_{i}+K_{i}^{t h}-A c_{i} c_{v}-p_{i} v_{i} c_{i}
$$

In eqs. (1) and (2) $\mathrm{Kf}_{\mathrm{V}}$ and $\mathrm{Kf} \mathrm{f}_{\mathrm{i}}$ are the rates of release of vacancies and interstitials, respectively, from :ascades into the medium with $K$ the total production rate of defect pairs. These terms take the place of the defect production rate in the usual rate equations. $K_{v}^{\text {th }}$ and $K_{i}^{\text {th }}$ are the thermal emission rates of vacancies and interstitials, respectively, from all sources including the clusters. $A$ is the recombination coefficient containing the jump frequencies $v_{V}$ and $v_{i}$ for vacancies and interstitials, and geometrical factors. $p_{v}$ and $p_{i}$ are the sink strengths, i. e., the probability per jump that the defect is annihilated at a sink. We will use throughout this paper "atomic units", i. e., the concentrations in eqs. (1) and (2) are in atomic fractions and the production rates $K$ and $K^{\text {th }}$ are numbers of defects/atom/s. It should be pointed out, that the sink terms in eqs. (1) and (2) are frequently expressed [21] as $\mathrm{Dk}^{2} \mathrm{c}$, instead of pvc, with $\mathrm{k}^{2}$ referred to as "sink strength" . Since the diffusion coefficient $D$ for defects in isotropic media equals $v b^{2} / 6$ with $b$ the jump or nearest neighbor distance, $k^{2}=6 \mathrm{p} / \mathrm{b}^{2}$. The meaning of $k^{2}$ is an inverse reaction cross section for defect annihilation per unit time, whereas $p$ is the probability that a defect is annihilated after its next jump.

For calculating the cluster size distributions we need to know the rates at which clusters of a given size are formed and are lost. The formation and loss can occur directly in cascades, or by shrinkage (or growth) of clusters of the next larger (or smaller) sizes. For calculating the rate of the latter process the "net flux" of defects in the medium is required:

$$
\Delta j=v_{i} c_{i}-v_{v} c_{v}
$$


Neglecting bias effects for simplicity $\left(p_{v}=p_{i}=p\right.$ ), we obtain from the steady-state solution of the rate equations (1) and (2):

$$
\Delta j=K\left(f_{i}-f_{v}\right) / p+\left(K_{i}^{t h}-K_{v}^{t h}\right) / p
$$

The growth or shrinkage rate of clusters [in number of defects/s] is obtained from the approximate solution of the diffusion equation for a spherical cluster of radius $\mathbf{r}_{\mathbf{n}}$ in the effective medium

$$
J\left(r_{n}\right)=\frac{2 \pi b^{2}}{3 \Omega} r_{n}\left(K\left(f_{i}-f_{v}\right) / p+\left(K_{i}^{t h}-K_{v}^{t h}\right) / p-\left(v_{i} c_{i}^{t h}\left(r_{n}\right)-v_{v} c_{v}^{t h}\left(r_{n}\right)\right)\right\}
$$

$\Omega$ is the atomic volume, $c_{v}^{\text {th }}\left(r_{n}\right)$ and $c_{i}^{\text {th }}\left(r_{n}\right)$, are the thermal equilibrium concentrations of vacancies and interstitials, respectively, at the surface of the cluster of $\mathbf{n}$ defects. The first term in the curly bracket of eq. (5) arises from the excess of mobile interstitials (over vacancies) escaping from cascades; the second term comes from the thermal emission of defects from all other sinks including clusters and from clusters becoming unstable by shrinkage; and the last term is the net defect loss by evaporation from the cluster of radius $r_{n}$ under consideration.

For fcc metals, $\Omega=b^{3 / 2}{ }^{1 / 2}$, and we obtain the shrinkage or growth rate in terms of the number of defects in the cluster:

$$
J(n)=(2 \pi)^{2 / 3} n^{1 / 3}\left\{K\left(f_{i}-f_{v}\right) / p+\left(K_{i}^{\text {th }}-K_{v}^{t h}\right) / p-\left(v_{i} c_{i}^{t h}(n)-v_{v} c_{v}^{\text {th }}(n)\right)\right\}
$$

Now we can write down the differential equations for the cluster size distribution for vacancies, $m_{v c l}(n)$. As we have discussed before, more interstitials than vacancies are likely to escape from clustering in cascades, making the first term in curly brackets of eq. (6) positive; the second term is, generally, negative, but smaller than the first; and the third term gives a positive contribution because interstitial evaporation is likely to be negligible. Hence, $J>0$, and vacancy clusters shrink, i. e. ,:

$$
\frac{\partial m_{v c l}(n)}{\partial t}=K_{v c l}(n)+J(n+1) m_{v c l}(n+1)-\left[J(n)+N_{d i s}(n) K_{c a s}\right] m_{v c l}(n)
$$

For $\mathrm{J}>0$ interstitial clusters grow, hence: 


$$
\frac{\partial m_{i c l}(n)}{\partial t}=K_{i c l}(n)+J(n-1) m_{i c l}(n-1)-\left[J(n)+N_{d i s}(n) K_{c a s}\right] m_{i c l}(n)
$$

$\mathrm{K}_{\mathrm{vcl}}(\mathrm{n})$ and $\mathrm{K}_{\mathrm{icl}}(\mathrm{n})$ are the rates at which vacancy and interstitial clusters of size $n$, respectively, are produced directly by cascades; $N_{\text {dis }}(n)$ is the number of sites within the cascade upon which a cluster of size $n$ can be centered and still be entirely dissolved in the "molten zone" of size $\mathrm{N}_{\text {cas }}$; and $\mathrm{K}_{\text {cas }}$ is the rate of cascade formation. The second term on the right-hand sides of eq. (7) (eq. (8)) is the gain of vacancy (interstitial) clusters of size $n$ by shrinkage (or growth) of clusters of the next larger (or smaller) size. The third terms are the losses by shrinkage (or growth) and by cascade dissolution.

The differential equations for the cluster size distributions can be integrated for any given set of irradiation and initial conditions. The sink strength, $p$, implicit in $J(n)$, must be recalculated to include the current cluster distributions for each integration step.

\section{Assumptions and input parameters for model calculations.}

To get some physical insights, calculations under a number of simplifying assumptions have been made:

1) All defects are produced by a single size of cascades characterized by the following quantities: the number of defect pairs produced by collisions, $\mathrm{N}_{0}$; the size of the "molten" region in which existing defect clusters are dispersed (expressed as number of sites affected, $N_{\text {cas }}$; we will assume that $\mathbf{N}_{\text {cas }}=f_{\text {cas }} N_{0}$, $i$. e., proportional to the cascade size); the fractions of vacancies, $f_{v}$, and interstitials, $f_{i}$, that escape from the cascade and the fraction, $f_{r}$, that recombine; the remaining interstitials form interstitial clusters of a single, small size $n_{i 0}$ in the peripheral regions of the cascade; the remaining vacancies are combined with the net number of defects resulting from dissolution of preexisting clusters and are assumed to form a single vacancy cluster of size $n_{\mathrm{vo}}$ in the central region of the cascade. The latter assumption derives from the picture that the molten zone solidifies epitaxially from its perimeter, depositing the net number of vacancies contained in the molten zone in a cluster.

With these assumptions and the conventional displacement rate, $K$, we have:

$$
\begin{aligned}
& \mathrm{K}_{\mathrm{cas}}=\mathrm{K} / \mathrm{N}_{\mathrm{O}} \\
& \mathrm{K}_{\mathrm{vcl}}\left(\mathrm{n}_{\mathrm{vo}}\right)=\mathrm{K}_{\mathrm{cas}} \text { and } \mathrm{K}_{\mathrm{vcl}}(\mathrm{n})=0 \text { for } \mathrm{n} \neq \mathrm{n}_{\mathrm{vo}}
\end{aligned}
$$


$\mathrm{K}_{\mathrm{icl}}\left(\mathrm{n}_{\mathrm{io}}\right)=\mathrm{K}_{\text {cas }} \mathrm{f}_{\mathrm{icl}} \mathrm{N}_{\mathrm{o}} / \mathrm{n}_{\mathrm{io}}$ and $\operatorname{Kicl}(\mathrm{n})=0$ for $\mathrm{n} \neq \mathrm{n}_{\mathrm{vo}}$

$n_{\mathrm{vo}}=f_{\mathrm{vcl}} N_{0}+\Sigma N_{\text {dis }}(n) n m_{\mathrm{vcl}}(n)-\Sigma N_{\text {dis }}(n) n m_{\text {icl }}(n)$,

with $f_{i c l}=1-f_{i}-f_{r}$ and $f_{v c l}=1-f_{V}-f_{r}$. The sums in equation (12) are extended over the existing size distributions of vacancy and interstitial clusters.

2) For cascade dissolution, the clusters are assumed to be circular dislocation loops with stacking faults. They are dissolved only if the entire loop is contained within the molten zone. For fcc lattices we obtain the following approximate expression:

$N_{\text {dis }}(n)=N_{\text {cas }}\left\{1-n^{1 / 2}(4 / \sqrt{3} \pi)^{1 / 6} / N_{\text {cas }}{ }^{1 / 3}\right\}^{3}$ for $n<n_{\text {imax }}$

$N_{\text {dis }}(n)=0 \quad$ for $n \geq n_{\text {imax }}$

3) The maximum size of interstitial loops subject to dissolution is determined from eq. (13) by $N_{\text {dis }}\left(n_{\text {imax }}\right)=0$. Interstitial loops that grow beyond that size are considered to become part of the dislocation network.

4) Vacancy loops that shrink below a size $n_{\text {vmin }}$ are assumed to dissolve spontaneously and the resulting vacancies, $\left(\mathrm{n}_{\mathrm{vmin}}{ }^{-1}\right) \mathrm{m}_{\mathrm{vcl}}\left(\mathrm{n}_{\mathrm{vmin}}\right) J\left(\mathrm{n}_{\mathrm{vmin}}\right)$, are added to the thermal production rate of vacancies.

5) The sink strength contributions of the clusters are calculated in the approximation of spherical sinks in an effective medium. Sink strengths for vacancy and interstitial clusters are corrected for the presence of the other sinks, $p$, by the factor $\left\{1+r(n) / b(6 p)^{1 / 2}\right\}$ with $r(n)$ the radius of the sink [20].

6) Thermal evaporation of defects from clusters is neglected. This has the advantage that none of the quantities calculated depend explicitly on the displacement $r$ te when compared on basis of dose. It should be noted, however, that the effects of thermal instability of small vacancy clusters can be explored by adjusting the minimum stable vacancy cluster size, $\mathbf{n}_{\mathrm{vmin}}$.

For the calculations, a set of reference values for the input parameters has been used. The values of several of them are rather uncertain; therefore, the sensitivity of the results to the choices were explored. Most of the reference parameter values were chosen to be consistent with values derived by Heinisch [9] 
from Marlowe type binary collision simulations of cascades in copper with subsequent "quenching" and "short term annealing": The calculations were performed with numbers of defects, $N_{0}=800,400,200,100,50$, corresponding to cascades with $60,30,15,7.5$ and $3.75 \mathrm{keV}$ of damage energy; the values of the fraction of vacancies escaping from large cascades $(>15 \mathrm{keV}), f_{v_{0}}=0.01$, and that of defects recombining, $f_{r o}=0.77$, are those of Heinisch. The $v_{\text {at }}$ ue for $f_{i 0}$ has been increased from the Heinisch value of 0.04 to 0.1 corresponding to a minimum stable and immobile interstitial cluster size of 5 at elevated temperatures which appears more consistent with significant growth of interstitial clusters during stage II annealing [22]. For the cascade size dependences of $f_{v}, f_{i}$ and $f_{r}$ we have assumed simple exponential forms,

$$
\begin{aligned}
& f_{v}=f_{v o} \exp \left(\left(1-N_{0}\right) / N_{d}\right) \\
& f_{i}=f_{i o} \exp \left(\left(1-N_{o}\right) / N_{d}\right) \\
& f_{r}=f_{r o}\left\{1-\exp \left(\left(1-N_{0}\right) / N_{d}\right)\right\},
\end{aligned}
$$

with a single decay parameter, $\mathrm{N}_{\mathrm{d}}$. Equation (14) with $\mathrm{N}_{\mathrm{d}}=40$ gives a reasonable representation of the simulation results by Heinisch [9].

The assumption of one big, immobile vacancy cluster, instead of many small clusters observed in the simulations by Heinisch, has been made on the basis of TEM observations (see, e. g., Black et al. [7], Kiritani et al. [8]) and some molecular dynamics simulations of cascades (see, e. g., Diaz de la Rubia et al. [19]).

The choice for the volume of the "molten" zone, $N_{\text {cas }}=f_{\text {cas }} N_{0}$, with $f_{\text {cas }}=15$ is rather uncertain, but seems reasonable in the light of scarce existing results from molecular dynamics simulations. As sink strength for the matrix $p_{0}=0.001$ was chosen as reference value; this sink strength is rather high, and corresponds to a disiocation density of approximately $10^{12} / \mathrm{cm}^{2}$. The minimum stable and immobile vacancy cluster size for the reference set is $n_{v o}=3$; as will be seen from the calculation, the particular choice of the value for $n_{\mathrm{vo}}$ has, within limits, little bearing on the results. The choice for the size of interstitial clusters at formation, $n_{i 0}=5$, again has little effect on the results. As already discussed, $n_{v c l}=1$, i. e., one vacancy cluster per cascade. Although the calculations were performed with a displacement rate of $K=10^{-3} \mathrm{dpa} / \mathrm{s}$, the results are independent of the rate as long as thermal processes are not explicitly taken into account and states are compared on the basis of dose rather than time.

All calculations were done with $f_{i}>f_{v}$, i. e., a larger fraction of mobile interstitials escapes from cascades at formation than vacancies. This implies that the 
fraction of interstitials that form clusters in cascades is smaller than the corresponding fraction for vacancies, $f_{i c l}<f_{v c l}$.

\section{Results of model calculations.}

The results of the calculations were very similar for all cascade sizes in regard to the evolution of the cluster distributions, sink strength and diffusior: characteristics. Therefore, we will illustrate the major characteristics by the results from one cascade size. Figure 1 shows the evolution of the size distributions for vacancy and the interstitial clusters obtained with the reference values of the input parameters just discussed and the single cascade size $N_{0}=200$ (15 keV). The maxima in the cluster concentrations occur at sizes of formation for both the vacancy and the interstitial clusters. The distributions "fill in" with increasing dose starting from the size of formation as expected. For vacancy clusters, the distribution at $1 \mathrm{dpa}$ (points not plotted in the graph) is very close to that of the quasy-steady state distribution shown. Similarly, the interstitial cluster distributions approach the quasi-steady state values rather rapidly at small sizes. However, it takes about 3.5 dpa for significant numbers of interstitial loops to grow to sizes exceeding the cascade dissolution limit ( 230 defects in this case). The form of the distributions and the approach to quasi-steady state are a consequence of cluster growth (or shrinkage) in presence of cascade dissolution: longer times are required to grow interstitial clusters to larger sizes (or in case of vacancy clusters to shrink to smaller sizes ), hence, the more clusters of sizes further removed from the size of formation are lost by cascade dissolution. The character of the distributions and their evolution are similar for different cascade sizes. The major distinction is size of vacancy clusters at their formation and, therefore, the location of the peak in the vacancy cluster distribution which shifts to larger sizes for larger cascades and vice versa.

The sink strength resulting from the cluster distributions are dominated by the high concentrations of clusters near their size of formation. The total sink strength, and those arising from matrix sinks, interstitial clusters and vacancy clusters are shown in Fig. 2 as function of dose. After an initial strong increase, the sink strength continues to increase at a rather modest rate. It is apparent that the matrix sink strength, although chosen to be very high, contributes very little to the total sink strength after a rather small dose (less than $0.1 \mathrm{dpa}$ ). The largest contributions comes from the small interstitial clusters and, to a lesser degree, from the large vacancy clusters.

The effect of the defect clusters on diffusional processes is illustrated in Fig. 3. The average number of jumps defects make is plotted as function of dose for 15 $\mathrm{keV}$ cascades $\left(\mathrm{N}_{0}=200\right)$. Reflecting the increase in cluster sink strength, the average number of jumps per defect decreases rapidly to about $0.2 \mathrm{dpa}$ and drifts 
only slightly to lower values for higher doses. It should be emphasized, that only diffusion jumps of defects escaping clustering and in-cascade recombination and, therefore, contribute to long range diffusion are included. Intra-cascade defect motion occurring during the cascade events that are important, e.g., to low temperature ion beam mixing, are not included. For comparison, the value obtained for in-cascade recombination, neglecting the cluster sinks, and that for defects created isolated as in electron irradiations are shown. It is obvious that the defect cluster sinks can reduce diffusion significantly from that predicted from incascade recombination. An important consequence of the larger fraction of interstitials escaping cascades than vacaucies is their correspondingly larger contribution to long range diffusion processes. This is at variance with the long held believe that interstitials and vacancies contribute equally to diffusion in the steady state approximation. The latter is valid only in the absence stable defect clusters.

The size of the central vacancy cluster in the cascade changes as a function of dose as a consequence of the difference in the number of interstitials and vacancies in clusters dissolved in the "molten" zone of the cascade. As shown in Fig. 4, initially the interstitials from the large number of small interstitial clusters contained entirely in the molten zone outnumber the vacancies from dissolved clusters, and the net number of vacancies available for formation of the new cluster decresises with dose. As interstitial clusters grow to larger sizes, that are less likely to be dissolved, and vacancy clusters shrink to smaller sizes, that are more likely to be dissolved, the difference in the number of dissolved defects decreases, and the size of the vacancy clusters at formation starts to increase towards higher doses. This non-monotonic change in $\mathrm{N}_{0}$ is the reason for the existence of a small concentration of vacancy clusters at sizes larger than that of the peak concentration in Fig. 1.

Variations of the input parameters affect, of course, the details of the calculated cluster distributions and sink strengths. However, the general features are surprisingly robust against variations of the input parameters over the entire reasonable range. As an example, the effect of changing the fraction of interstitials escaping from cascades, $f_{i o}$, over the range from 0.05 to 0.15 on the total sink strength and the contributions by interstitial and by vacancy clusters is shown in Fig. 5 for $15 \mathrm{keV}$ cascades. A factor of 3 in $\mathrm{f}_{\mathrm{io}}$ causes less than $20 \%$ changes in the total sink strength over most of the dose range shown. Changes in $f_{\mathrm{vo}}$, the fraction of vacancies escaping into the matrix, show similarly modest changes in the calculated quantities. As one would expect, the matrix sink strength has little effect because of the dominance of the cluster sinks.

The effect of the assumed minimum stable vacancy cluster size, $n_{y m i n}$, has been explored and some results are presented in Figure 6 . An increase of $n_{v m i n}$ from 3 
to 25 vacancies decreases the total sink strength by about $15 \%$. The vacancy clusters in this case are formed at size 30 . However, any further increase in the stable minimum size causes a rapid drop in the cluster populations and, therefore, in the sink strength. Generally, cluster size distributions are little affected by $\mathbf{n}_{\text {ymin }}$ as long as it remains smaller than about $80 \%$ of the vacancy cluster size at formation. This result indicates that the neglect of thermal emission from clusters should be a reasonable assumption up to temperatures where the vacancy clisters at the size of their formation in cascades are thermally stable.

\section{Summary and conclusions.}

Clusters formed in cascades develop at temperatures between the annealing stages III and V under most circumstances to be the dominant sinks for mobile point defects after a fraction of a displacement per atom.

Dissolution of clusters by cascades is important in attaining quasi-steady-state cluster populations. As a consequence, the distribution of cluster sizes peak at the size of formation, i. e., for vacancy clusters at the large end and for interstitial clusters at the small end.

This leads to important secondary consequences:

(a) the thermal dissolution of vacancy clusters contributes negligibly to the mobile vacancy concentration except when the size of instability approaches the size of formation.

(b) the rate of production of interstitial dislocation loops exceeding the dissolution size limit by cascades is rather low initially, but becomes significant after a dose of several displacements per atom (about $3-4 \mathrm{dpa}$ ).

Although a smaller fraction of interstitials cluster, interstitial clusters contribute generally more to the sink strength than vacancy clusters. As a consequence of more interstitials than vacancies escaping into the matrix, the contribution of interstitials to long range diffusion is larger than that of vacancies, in contrast to the case when in-cascade clustering is neglected.

As a consequence of the large sink strength of defect clusters produced under irradiation conditions in which significant numbers of large cascades occur, the steady state concentration of defects is reduced significantly below that in which few or no stable defect clusters are produced. Therefore, rate theory predictions neglecting the presence of defect clusters formed in cascades grossly overestimate many phenomena that are controlled by radiation-enhanced diffusion or radiation-induced segregation. 
[1] W. Schilling, G. Burger, K. Isebeck and $H$. Wenzel in: Vacancies and Interstitials in Metals, A. Seeger, D. Schumacher, W. Schilling and J. Diehl, eds., North Holland, Amsterdam, 1970, p.255

[2] R. S. Averback, R. Benedek and K. L. Merkle, Phys. Rev. B 18 (1978) 4156

[3] L. E. Rehn, P. R. Okamoto and R. S. Averback, Phys. Rev. B30(1984)3073

[4] M.-P. Macht, A. Müller, V. Naundorf and H. Wollenberger

Nucl. Instr. and Meth. B 16 (1986) 148

[5] L. E. Rehn and P. R. Okamoto, Materials Science Forum 15-18 (1987) 985

[6] A. Müller, M.-P. Macht and V. Naundorf, J. Nucl. Mater. J. Nucl. Mater. (1990) in press (Proc. ICFRM-4, Kyoto, 1989)

[7] J. Black, M. L. Jenkins, C. A. English and M.A. Kirk, Proc. R. Soc. Lond. A409 (1987) 177

[8] M. Kiritani, T. Yoshiie, S. Kojima and Y. Satoh, Rad. Eff. Def. Sol.113(1990)75, M. Kiritani, Materials Science Forum, 15-18 (1987) 1023

[9] H. L. Heinisch, Rad. Eff. Def. Sol.113 (1990) 53

[10] H. Wiedersich, Rad. Eff. Def. Sol. 113 (1990) 97, J. Nucl. Mater. 179/181(1991) in press (Proc. ICFRM-4, Kyoto, 1989)

[11] $\mathrm{H}$. Wiedersich, Rad. Effects 12 (1972) 111

[12] R. Bullough, B. L. Eyre and K. Krishan, Proc. Royal Soc. A346(1975)81

[13] R. Bullough and T. M. Quigley, J. Nucl, Mater. 113 (1983) 179

[14] C. H. Woo and B. N. Singh, phys. stat. sol. (b) 159 (1990) 609

[15] H. Wiedersich, Nucl. Instr. Methods, B (1991), in press

[16] W. Schilling, P. Ehrhart and K. Sonnenberg in "Fundamental Aspects of Radiation Damage in Metals", M. T. Robinson and F. W. Young, Jr., eds.,US Energy Research and Development Administration, Conf.-751006, 1975, Vol.P1, p.470

[17] N. Yoshida, Y. Akashi, K. Kitajïma and M. Kiritani, J. Nucl. Mater. 133/134 (1985) 405

[18] M. W. Guinan and J. H. Kinney, J. Nucl. Mater. 103/104 (1981) 1319

[19] T. Diaz de la Rubia, R. S. Averback, R. Benedek and I. M. Robertson, Rad. Eff. Def. Sol.113 (1990) 39

[20] A. D. Brailsford and R. Bullough, Phil. Trans. Royal Soc. London 302 (1981) 87

[21] A. D. Brailsford and R. Bullough, J. Nucl. Mater. 44 (1972) 121

[22] P. Ehrhart, K. H. Robrock and H. R. Schober, in Physics of Radiation Effects in Crystals, R. A. Johnson and A. N. Orlov, eds., North-Holland, Amsterdam, Oxford, New York, Tokyo, 1986, pp. 3-115

Figure Captions. 


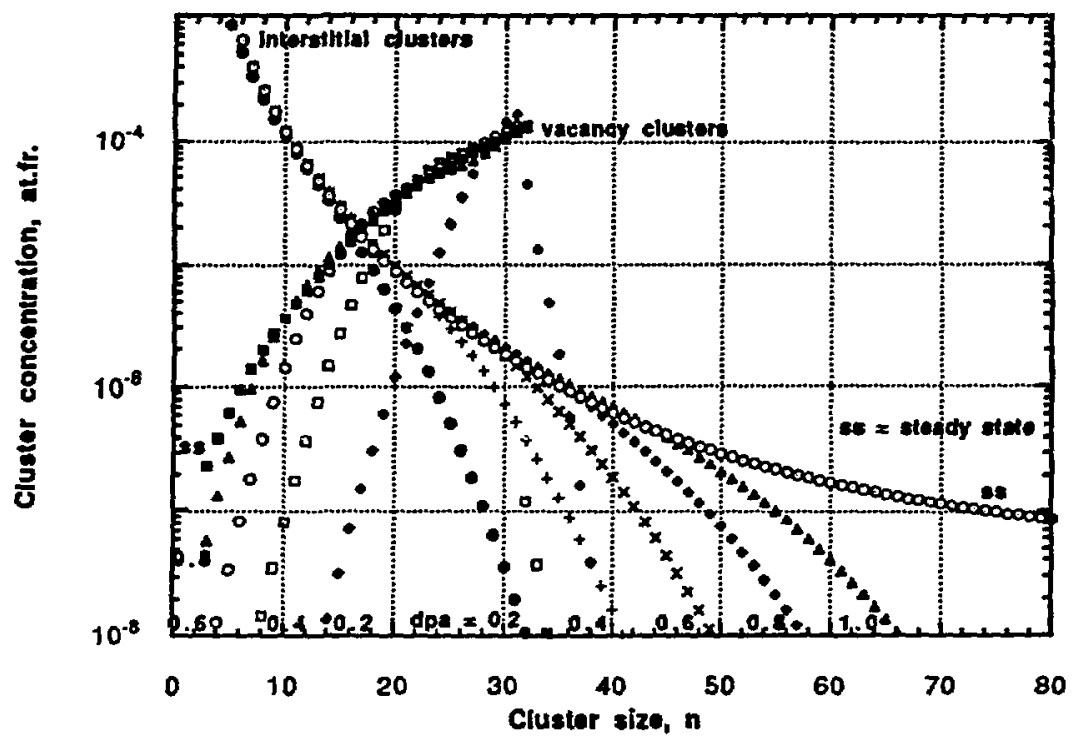

Fig. 1. Size distributions of vacancy and interstitial clusters for doses up to 1 dpa for $15 \mathrm{keV}$ cascades. $\mathrm{N}_{0}=200, \mathrm{f}_{\mathrm{vo}}=0.01, \mathrm{f}_{\mathrm{i} 0}=0.1, \mathrm{f}_{\mathrm{r}}=0.77, \mathrm{f}_{\text {cas }}=15, \mathrm{~N}_{\mathrm{d}}=$ 40. 


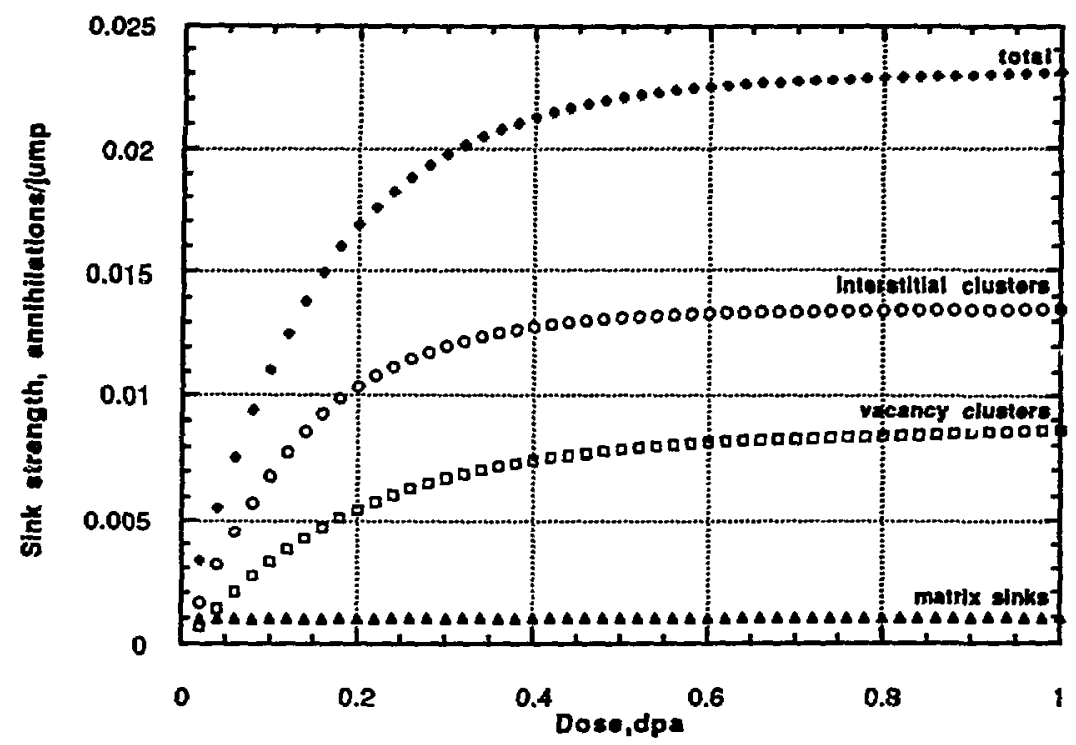

Fig. 2. Total sink strength and the contributions of matrix sinks, vacancy clusters and interstitial clusters as function of dose. $N_{0}=200, f_{v 0}=0.01, f_{10}=$ $0.1, f_{\mathrm{r}}=0.77, \mathrm{f}_{\text {cas }}=15, \mathrm{~N}_{\mathrm{d}}=40$.

(Parameters as in Fig.1.) 


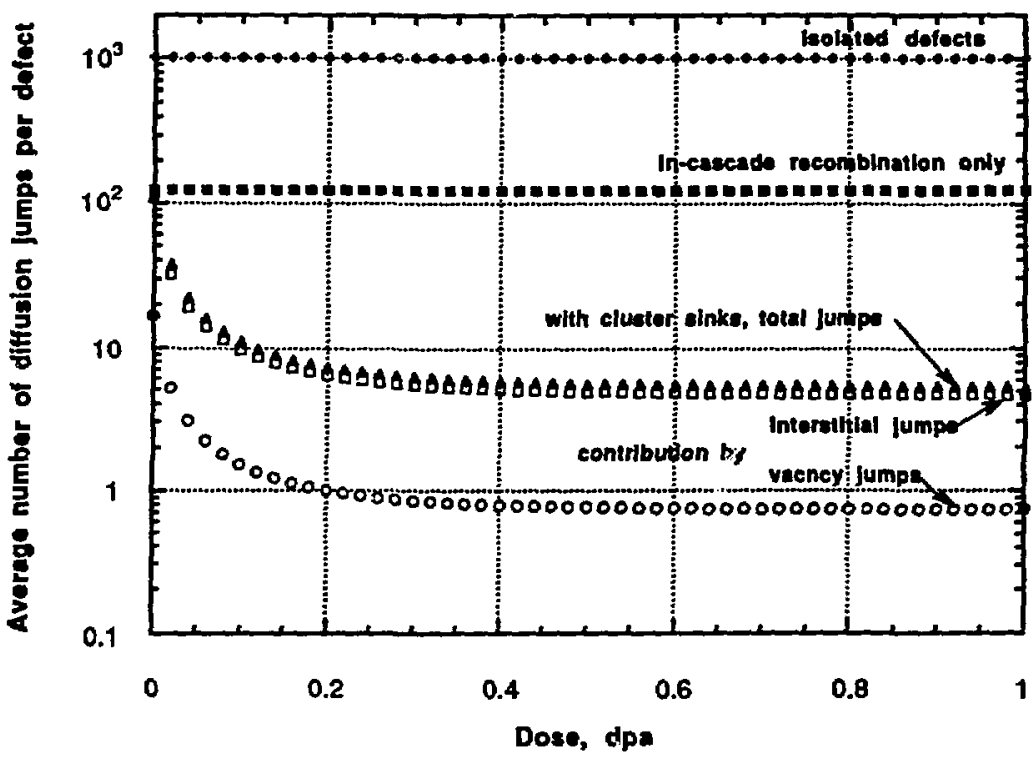

Fig. 3. Average number of diffusion jumps per defect produced in $15 \mathrm{keV}$ collision cascades as function of dose. Only thermal jumps of defects escaping in-cascade recombination and clustering are included. Shown are vacancy jumps, interstitial jumps, their sum, i.e., the total contribtion to diffusion of atoms. The value for the cases of in-cascade recombination without cluster sinks and for production of isolated defects are shown for comparison.

$N_{0}=200, f_{\text {vo }}=0.01, f_{\text {io }}=0.1, f_{\mathrm{r}}=0.77, f_{\text {cas }}=15, N_{d}=40$. 


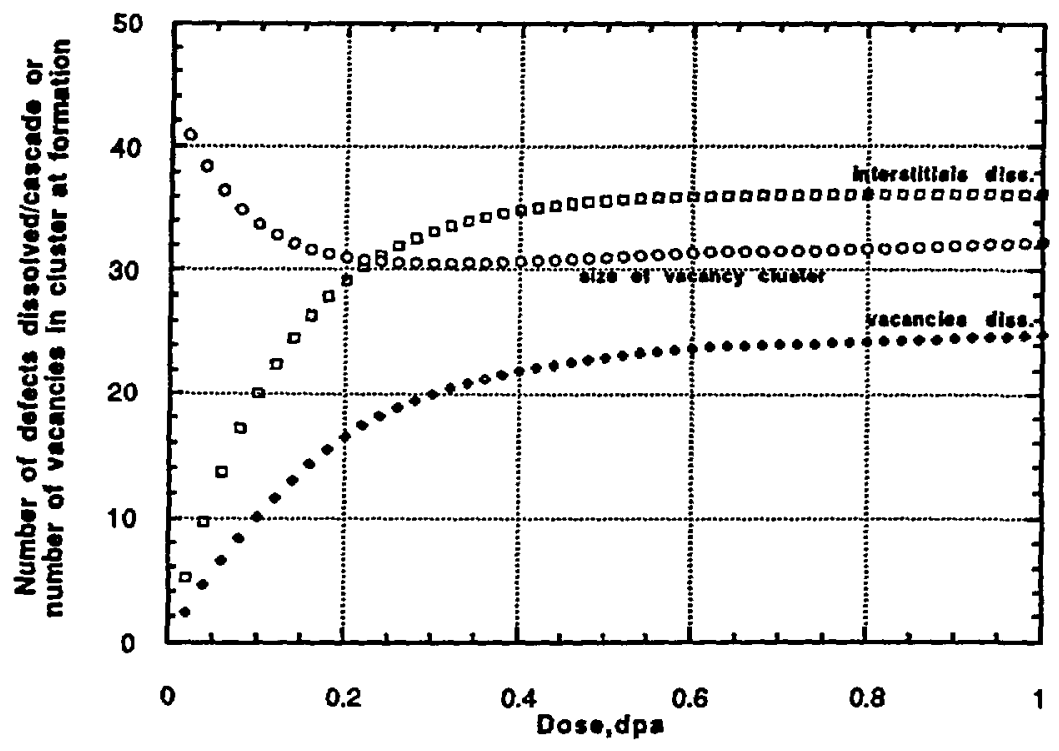

Fig. 4. Number of interstitials und of vacancies dissolved per cascade and net number vacancies in vacancy cluster formed by $15 \mathrm{kev}$ cascades as function of dose. $N_{o}=200, f_{v o}=0.01, f_{i 0}=0.1, f_{r}=0.77, f_{\text {cas }}=15, N_{d}=40$. 


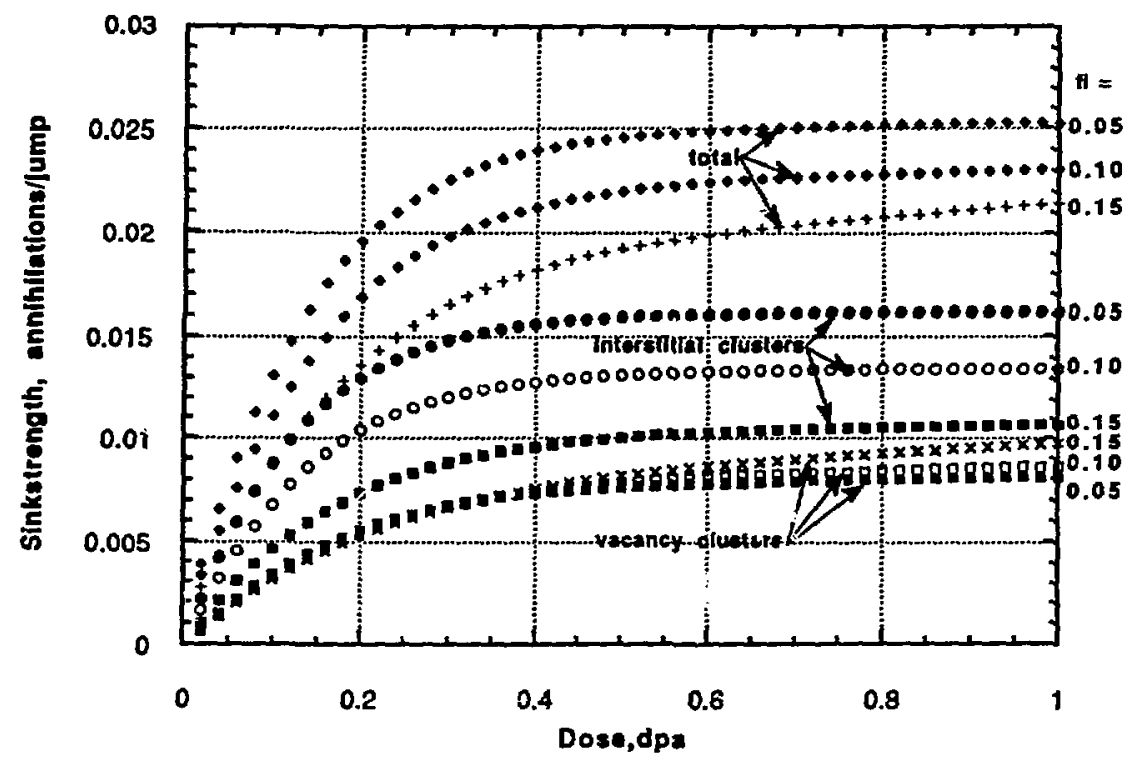

Fig. 5. Effect of the fraction of interstitials escaping from in-cacade recombination and clustering on the total sink strength and that of interstitial and vacancy clusters as function of dose. $f_{i o}=0.05,0.10$ and $0.15 ; N_{0}=200, f_{v o}=0.01, f_{r}=0.77, f_{c a s}=15, N_{d}=40$. 


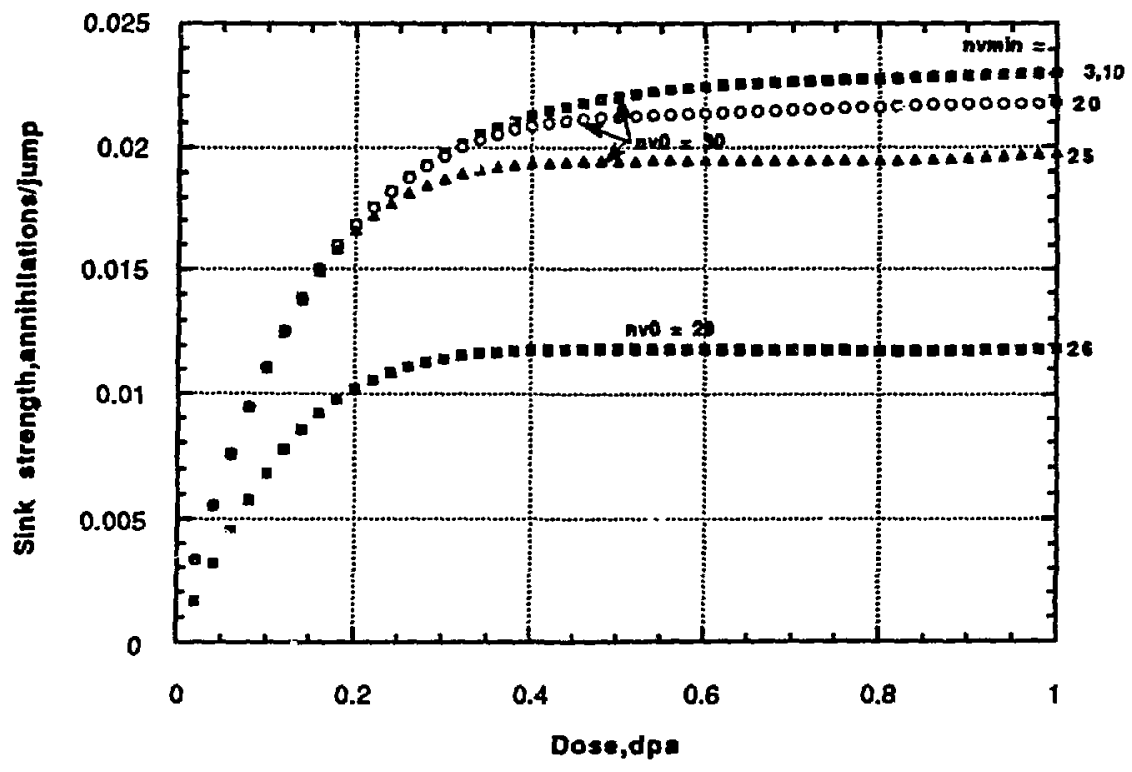

Fig. 6. Effect of the minimum stable vacancy cluster size on the development of the total sink strength. The size of formation near the minimum of nvo, see Fig. 4, is 30 vacancies for $n v m i n=3,10,20$ and 25 , and 29 vacacancies for nvmin $=26$. For larger nvmin's the formation size drops below the minimum stable size. $f_{i 0}=0.05,0.10$ and $0.15 ; N_{0}=200, f_{v o}=0.01$, $f_{\mathrm{r}}=0.77, f_{\text {cas }}=15, N_{d}=40$. 